\title{
Different Approaches to the Modelling of COVID-19
}

\author{
J. F. C. A. MEYER ${ }^{1}$, M. LIMA² , C. C. ESPITIA ${ }^{3}$, F. LONGO ${ }^{4}$, B. LAIATE ${ }^{5}$, \\ A. N. GOIS ${ }^{6}$ and C. F. D. KUNZ
}

Received on August 29, 2020 / Accepted on March 16, 2021

\begin{abstract}
In this paper some innovative aspects of the mathematical modelling of classic epidemiology problems for the study of models related to the COVID-19 pandemic dynamics are presented. In addition, they are compared to real-world data using numerical methods in order to approximate the solutions. One of these models includes a non-transmitting compartment and another one, a delay-differential equation in the SIR-type method. Finally, a comparative discussion of the results is also presented.
\end{abstract}

Keywords: COVID-19, mathematical modelling, basic reproductive number, mathematical epidemiology, nonlinear systems of ODE.

\section{INTRODUCTION}

Many texts have been made public in order to contribute to a better understanding and an increased knowledge about the dynamic behaviour of this new disease, the COVID-19.

The historical aspects of its appearance (in spite of intriguing results of the presence of the SARS$\mathrm{CoV}-2$ in sewers in places where the disease was only identified much later [11,14]) will surely have been described objectively in many of the introductions of the texts published in this very

\footnotetext{
*Corresponding author: J. F. C. A. Meyer - E-mail: jmeyer@unicamp.br

${ }^{1}$ Department of Applied Mathematics, IMECC, University of Campinas, R. Sérgio Buarque de Holanda, 651, 13083-859, Campinas, SP, Brazil - E-mail: jmeyer@unicamp.br https://orcid.org/0000-0001-8002-7075

2 Department of Applied Mathematics, IMECC, University of Campinas, R. Sérgio Buarque de Holanda, 651, 13083-859, Campinas, SP, Brazil - E-mail: marina@ime.unicamp.br https://orcid.org/0000-0002-8520-4306

3 Department of Applied Mathematics, IMECC, University of Campinas, R. Sérgio Buarque de Holanda, 651, 13083-859, Campinas, SP, Brazil - E-mail: espitiacristian@gmail.com https://orcid.org/0000-0001-5500-0460

${ }^{4}$ Department of Applied Mathematics, IMECC, University of Campinas, R. Sérgio Buarque de Holanda, 651, 13083-859, Campinas, SP, Brazil - E-mail: longo@ime.unicamp.br https://orcid.org/0000-0002-2868-3937

5 Department of Applied Mathematics, IMECC, University of Campinas, R. Sérgio Buarque de Holanda, 651, Campinas, SP, 13083-859, Brazil - E-mail: beatrizlaiate@gmail.com https://orcid.org/0000-0002-7312-4843

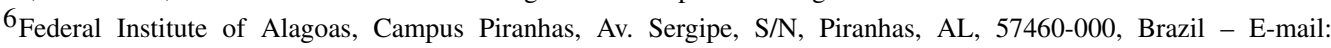
aedson.gois@gmail.com https://orcid.org/0000-0002-9118-6247

${ }^{7}$ Frankfurt Institute for Advanced Studies, Goethe University Frankfurt, Ruth-Moufang-Straße 1, 60438, Frankfurt am Main, Germany - E-mail: camileknz@gmail.com https://orcid.org/0000-0002-7193-9002
} 
number of the journal TEMA - Trends in Applied and Computational Mathematics. As well as in so many other academic publications $[1,3,7,12,16,17,19]$.

In view of these considerations, in this introductory part of our work we would like to highlight the mathematical modelling efforts that have been undertaken for a long time, with certain emphasis in the beginning of the twentieth century. An additional difficulty has always been centered on the considered dependent variables and the adequate parameters in the nonlinear systems of Ordinary Differential Equations (ODEs) and Partial Differential Equations (PDEs).

It is evident that each of the mentioned models have their many multiple characteristics and purposes, but precise quantitative forecasts are many times not one of these aspects and objectives, since relevant conclusions on the qualitative behaviors are also very necessary in the study of epidemics and in the understanding of the model and its modeled phenomena. Besides, the everpresent challenge of moving on to an improved model with which serious and useful work can be meaningful.

An historical mention to [9] is mandatory as is the preference of the authors to the simplicity of which can be useful whereas the tendency to those so-called 'spaghetti models' with its possibly dubious correlations among variables (dependent and independent) should be avoided.

In this work we will discuss the use of some models which we believe may contribute positively to a better effort in evaluating public policies in order to be able to plan the reversal of a still disastrous sanitary and social situation worldwide.

In order to include the incubation period of the SARS-CoV-2 [21], two modelling options were considered: one was to create a specific compartment for individuals in this situation (see Subsections $2.3,2.5,2.6$ ), while the second one was introducing a time-period in which asymptomatic individuals still do not transmit the virus (see Subsection 2.4). Thus, we will, successively, present and comment, in some simplicity, classic and new models, which we have named according to a traditional use: SIRD, SEIRD, SCAIRD, SCEIRD and SCEAIRD, with a special characteristic in one of them (the SCAIRD model), due to the mentioned use of a delaydifferential equation for one of the modeled aspects. As described before, this aspect consists on the contagion through the contact of a still asymptomatic individual after a certain period of time [21].

Lastly, we did not approach the study of those models which include many more compartments, as presented in an instructive paper [13] in which three different compartments describe three quite different situations for individuals in hospital situations. This is not due to the complexity of this type of model. Rather, this is due to the purpose of our academic effort in beginning with the simpler situations. We emphasize that the models presented do not consider population dynamics.

The manuscript is organized as follows: In Section 2, we recover the classic SIR epidemiological model and propose five new models for COVID-19: SIRD, SEIRD, SCAIRD, SCEIRD and SCEAIRD, and for each one, we study the local stability of the disease free equilibrium in terms 
of the basic reproduction number $\left(R_{0}\right)$; in Section 3 we perform simulations for the proposed models and we compute the $R_{0}$ of the COVID-19 system model and we discuss about the usefulness of our model, where we use real data from Brazil. We end this work with Section 4 of conclusions and future research.

\section{COVID-19 MODELS}

In this section we will very briefly mention simpler models we have used in order to compare results for those with original aspects, which are described in sequence.

\subsection{The Classic SIR Epidemiological Model}

This is, of course, the classic Kermack and McKendrick Model [9], with which we establish a behaviour for gauging the dynamics of the models we will used here. The traditional compartments are given by $S$ : the Susceptible, $I$ : the Infected and $R$ : the Recovered population.

The ability with which a virus spread over a population is measured by the most important number in an epidemiological model called basic reproduction number, which is denoted by $R_{0}$. It represents "the expected number of secondary cases produced in a completely susceptible population by a typical infected individual". If $R_{0}<1$ then one infected individual can infect less than one new infection over the course of its infectious period, and the disease can not grow. However if $R_{0}>1$ then one infected individual produces more than one new infection, and thus the disease invades the population and it is considered an epidemic [5].

Many models have a disease free equilibrium (DFE) at which the population remains in the absence of disease, and many researches use this point to calculate $R_{0}$ as the spectral radius of the next generation matrix, for more details we recommend Van den Driessche [18]. Thus, to determine the basic reproduction number, for the models in this work, the next generation method developed by Van den Driessche is used, and for the present model with time delay this basic number is calculated according the characteristic polynomial of the Jacobian matrix as described in [8].

\subsection{The SIRD Model}

In this model, we consider the following classes of individuals: $S$ (Susceptible), $I$ (Infected), $R$ (Recovered - with temporary immunity) and $D$ (Dead), see Figure 1.

Model parameters: $\alpha_{S}$ : transmission coefficient from the compartment of susceptible to that of infected individuals; $\gamma_{I}$ : recovery rate of infected individuals; $\mu_{I}$ : death rate of infected individuals; and $\eta$ : rate of immunity loss. The initial conditions this and the following ODE systems are: $S(0)=$ total population of Brazil $-1, I(0)=1, R(0)=0$ and $D(0)=0$.

Furthermore, we do not consider vital dynamics in this case, that is,

$$
S(t)+I(t)+R(t)+D(t)=N=\text { constant } .
$$




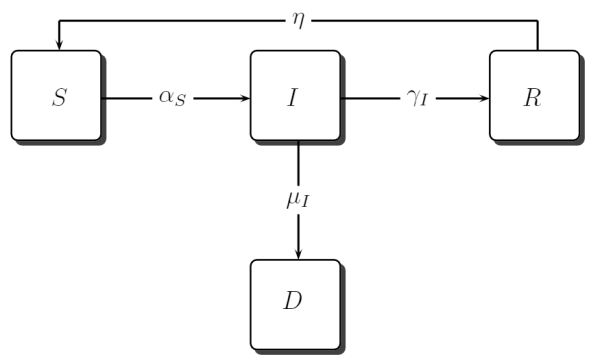

Figure 1: Diagram for SIRD model

The SIRD model can be writen as:

$$
\begin{aligned}
\frac{d S(t)}{d t} & =-\alpha_{S} S(t) I(t)+\eta R(t) \\
\frac{d I(t)}{d t} & =\alpha_{S} S(t) I(t)-\gamma_{I} I(t)-\mu_{I} I(t) \\
\frac{d R(t)}{d t} & =\gamma_{I} I(t)-\eta R(t) \\
\frac{d D(t)}{d t} & =\mu_{I} I(t)
\end{aligned}
$$

The only infected population is in the compartment $I(t)$, and we assume that the disease-free equilibrium, DFE, can only be $\left(S_{0}, 0,0,0\right)$, thus:

$$
R_{0}=\frac{\alpha_{S} S_{0}}{\gamma_{I}+\mu_{I}}
$$

The basic reproductive number (2.1) is exactly the same as that of the SIR model, as it was expected. SIRD model is almost the same as the SIR model, except for the use of a compartment for dead individuals, which was meant to help calibrate parameters, although the sub-notification in deaths is also widely observed worldwide.

\subsection{The SEIRD Model}

The purpose of this model is to be able to include a new compartment for those individuals that, after being exposed to the virus and receiving it, are not yet its transmitters. So, in this model, we consider the following classes of individuals: $S$ (Susceptible), $E$ (Exposed - individuals who contracted the virus, but do not yet infect others, nor manifest symptoms), I (Infected - individuals who transmit the virus), $R$ (Recovered - with temporary immunity) and $D$ (Dead), as shown in Figure 2. One can observe that Exposed and Infected individuals are all infected, but belong in different compartments, or sub-compartments.

Model parameters: $\alpha_{S}$ : infection coefficient from susceptible to exposed individuals, who acquire the virus but still do not infect others; $\alpha_{E}$ : rate of exposed individuals who start to manifest 


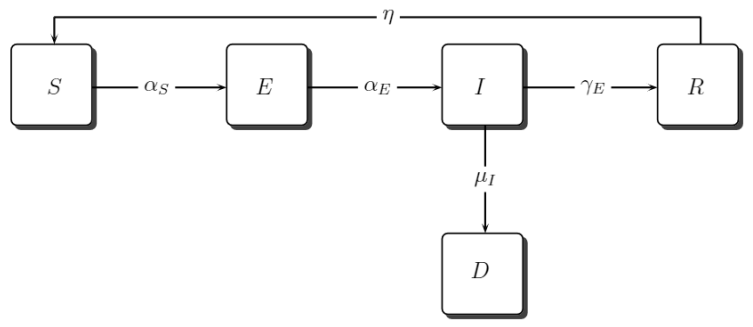

Figure 2: Diagram for SEIRD model

symptoms and begin to be considered as infected, that is, they may infect others; $\gamma_{E}$ : recovery rate of infected individuals; $\mu_{I}$ : death rate of infected individuals; and $\eta$ : rate of immunity loss. The initial conditions of the ODE systems are: $S(0)=$ total population of Brazil $-1, E(0)=0$, $I(0)=1, R(0)=0$ and $D(0)=0$.

Furthermore, we do not consider vital dynamics in this case, that is,

$$
S(t)+E(t)+I(t)+R(t)+D(t)=N=\text { constant }
$$

The SEIRD model can be writen as:

$$
\begin{aligned}
\frac{d S(t)}{d t} & =-\alpha_{S} S(t) I(t)+\eta R(t) ; \\
\frac{d E(t)}{d t} & =\alpha_{S} S(t) I(t)-\alpha_{E} E(t) ; \\
\frac{d I(t)}{d t} & =\alpha_{E} E(t)-\gamma_{I} I(t)-\mu_{I} I(t) ; \\
\frac{d R(t)}{d t} & =\gamma_{I} I(t)-\eta R(t) ; \\
\frac{d D(t)}{d t} & =\mu_{I} I(t) .
\end{aligned}
$$

Considering the DFE $\left(S_{0}, 0,0,0,0\right)$, according the next generation method let be $x=(E, I)$. We then have

$$
\begin{gathered}
\mathscr{F}(x)=\left(\begin{array}{c}
\alpha_{S} S I \\
0
\end{array}\right), \mathscr{V}(x)=\left(\begin{array}{c}
\alpha_{E} E \\
-\alpha_{E} E+\left(\gamma_{I}+\mu_{I}\right) I
\end{array}\right), F=\left(\begin{array}{cc}
0 & \alpha_{S} S_{0} \\
0 & 0
\end{array}\right) \\
V=\left(\begin{array}{cc}
\alpha_{E} & 0 \\
-\alpha_{E} & \gamma_{I}+\mu_{I}
\end{array}\right), V^{-1}=\left(\begin{array}{cc}
\frac{1}{\alpha_{E}} & 0 \\
\frac{1}{\gamma_{I}+\mu_{I}} & \frac{1}{\gamma_{I}+\mu_{I}}
\end{array}\right), F V^{-1}=\left(\begin{array}{cc}
\frac{\alpha_{S} S_{0}}{\gamma_{I}+\mu_{I}} & \frac{\alpha_{S} S_{0}}{\gamma_{I}+\mu_{I}} \\
0 & 0
\end{array}\right) .
\end{gathered}
$$

And, thus

$$
R_{0}=\frac{\alpha_{S} S_{0}}{\gamma_{I}+\mu_{I}}
$$

that is, the same value as in the SIRD model. 


\subsection{The SCAIRD Model}

In this model, we consider the time-lag from when an individual contracts the virus and the instant of time this individual may transmit it. Thus, the following classes of individuals are regarded: $S$ (Susceptible), $C$ (Confined - susceptible individuals under social distancing regime), $A$ (Asymptomatic - who do not have symptoms, but can transmit the virus), $I$ (Infected - symptomatic individuals who transmit the virus), $R$ (Recovered - with temporary immunity) and $D$ (Dead), see Figure 3. Here, it must be mentioned that Asymptomatic and Infected individuals are all infected, but in different compartmental classes, or sub-compartments.

As stated before, we consider a time period during which the asymptomatic individuals do not transmit the virus. For this, we will resort to some of the equations of the system with a retard term. A similar approach has been taken in previous works when incorporating delays resulting from the natural history of the infection [10]. As in the previous models, we do not consider vital dynamics in this case, that is,

$$
S(t)+C(t)+A(t)+I(t)+R(t)+D(t)=N=\text { constant } .
$$

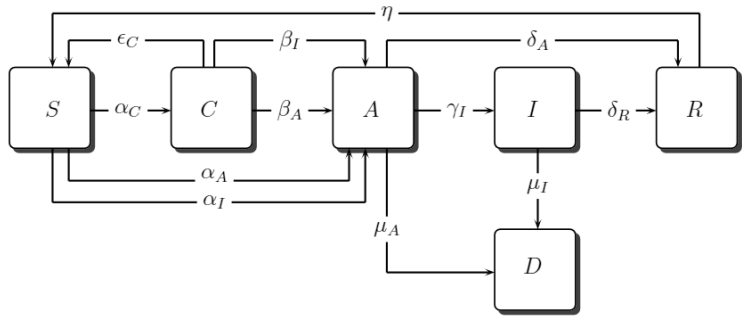

Figure 3: Diagram for SCAIRD model

Model parameters: $\alpha_{C}$ : rate of susceptible individuals that start being confined; $\alpha_{A}$ : transmission coefficient from the compartment of susceptible to that of asymptomatic individuals that contract the virus and become asymptomatic; $\alpha_{I}$ : transmission coefficient from susceptible to asymptomatic individuals that contract the virus and become infected; $\varepsilon_{C}$ : rate of confined individuals that leave, for some reason, the social distancing and, therefore, the confinement compartment; $\beta_{A}$ : transmission coefficient from confined to asymptomatic individuals that contract the virus and become asymptomatic; $\beta_{I}$ : transmission coefficient from confined to infected individuals that contract the virus and become asymptomatic; $\gamma_{I}$ : rate of asymptomatic individuals that become infected individuals; $\delta_{A}$ : recovery rate of asymptomatic individuals who do not manifest the disease nor infect others; $\delta_{R}$ : recovery rate of infected individuals; $\mu_{A}$ : death rate of asymptomatic individuals; $\mu_{I}$ : death rate of infected individuals; and $\eta$ : rate of immunity loss. The initial conditions of the ODE systems are: $S(0)=$ total population of Brazil $-1, C(0)=0$, $A(0)=0, I(0)=1, R(0)=0$ and $D(0)=0$. 
The SCAIRD model can be written as:

$$
\begin{aligned}
\frac{d S(t)}{d t}= & \eta R(t)-\alpha_{A} S(t) A(t-\tau)-\alpha_{I} S(t) I(t)-\alpha_{C} S(t)+\varepsilon_{C} C(t) \\
\frac{d C(t)}{d t}= & \alpha_{C} S(t)-\beta_{A} C(t) A(t-\tau)-\beta_{I} C(t) I(t)-\varepsilon_{C} C(t) \\
\frac{d A(t)}{d t}= & \alpha_{A} S(t) A(t-\tau)+\alpha_{I} S(t) I(t)+\beta_{A} C(t) A(t-\tau)+\beta_{I} C(t) I(t) \\
& -\gamma_{A} A(t)-\delta_{A} A(t)-\mu_{A} A(t) \\
\frac{d I(t)}{d t}= & \gamma_{A} A(t)-\delta_{R} I(t)-\mu_{I} I(t) \\
\frac{d R(t)}{d t}= & \delta_{A} A(t)+\delta_{R} I(t)-\eta R(t) \\
\frac{d D(t)}{d t}= & \mu_{I} I(t)+\mu_{A} A(t) .
\end{aligned}
$$

Considering the DFE $E_{0}=\left(S_{0}, 0,0,0,0,0\right)$, the characteristic polynomial of Jacobian evaluated at $E_{0}$ will be calculated from:

$$
J\left(E_{0}\right)=\left(\begin{array}{cccccc}
-\alpha_{C} & \varepsilon_{C} & -\alpha_{A} S_{0} & -\alpha_{I} S_{0} & \eta & 0 \\
\alpha_{C} & -\varepsilon_{C} & 0 & 0 & 0 & 0 \\
0 & 0 & \alpha_{A} S_{0}-\left(\gamma_{A}+\delta_{A}+\mu_{A}\right) & \alpha_{I} S_{0} & 0 & 0 \\
0 & 0 & \gamma_{A} & -\left(\delta_{R}+\mu_{I}\right) & 0 & 0 \\
0 & 0 & \delta_{A} & \delta_{R} & -\eta & 0 \\
0 & 0 & \mu_{A} & \mu_{I} & 0 & 0
\end{array}\right) .
$$

The characteristic polynomial is

$$
\begin{aligned}
p(\lambda)= & \lambda(\lambda+\eta)\left[\lambda^{2}+\lambda\left(\varepsilon_{C}+\alpha_{C}\right)+\varepsilon_{C}\left(\alpha_{A}+\alpha_{C}\right)\right] \\
& {\left[\lambda^{2}+\left(-S_{0} \alpha_{A}+\gamma_{A}+\delta_{A}+\delta_{R}+\mu_{I}+\mu_{A}\right) \lambda\right.} \\
& \left.+\left(\left(\delta_{R}+\mu_{I}\right)\left(\gamma_{A}+\delta_{A}+\mu_{A}\right)-S_{0}\left(\alpha_{I} \gamma_{A}+\alpha_{A}\left(\delta_{R}+\mu_{I}\right)\right)\right)\right] \\
\Rightarrow p(\lambda)= & \lambda(\lambda+\eta)\left[\lambda^{2}+\lambda\left(\varepsilon_{C}+\alpha_{C}\right)+\varepsilon_{C}\left(\alpha_{A}+\alpha_{C}\right)\right] \\
& {\left[\lambda^{2}+\left(-S_{0} \alpha_{A}+\gamma_{A}+\delta_{A}+\delta_{R}+\mu_{I}+\mu_{A}\right) \lambda\right.} \\
& \left.+\left(\left(\delta_{R}+\mu_{I}\right)\left(\gamma_{A}+\delta_{A}+\mu_{A}\right)\right)\left(1-\frac{S_{0}\left(\alpha_{I} \gamma_{A}+\alpha_{A}\left(\delta_{R}+\mu_{I}\right)\right)}{\left(\delta_{R}+\mu_{I}\right)\left(\gamma_{A}+\delta_{A}+\mu_{A}\right)}\right)\right]
\end{aligned}
$$

Therefore if, $\gamma_{A}+\delta_{A}+\delta_{R}+\mu_{I}+\mu_{A}>S_{0} \alpha_{A}$, the basic reproduction number is:

$$
R_{0}=\frac{\left[\alpha_{I} \gamma_{A}+\alpha_{A}\left(\delta_{R}+\mu_{I}\right)\right] S_{0}}{\left(\delta_{R}+\mu_{I}\right)\left(\gamma_{A}+\delta_{A}+\mu_{A}\right)} .
$$




\subsection{The SCEIRD Model}

A somewhat different approach to what was added in Subsection 2.4 is considered here adding a new compartment to the model, that of non-infective individuals that have received the virus. We then consider the following classes of individuals: $S$ (Susceptible), $C$ (Confined - susceptible individuals under social distancing regime), $E$ (Exposed - individuals who contracted the virus, but do not yet infect, nor manifest symptoms), $I$ (Infected), $R$ (Recovered - with temporary immunity) and $D$ (Dead), see Figure 4. We can observe that Exposed and Infected individuals are all infected, but belong to different compartmental classes, or sub-compartments.

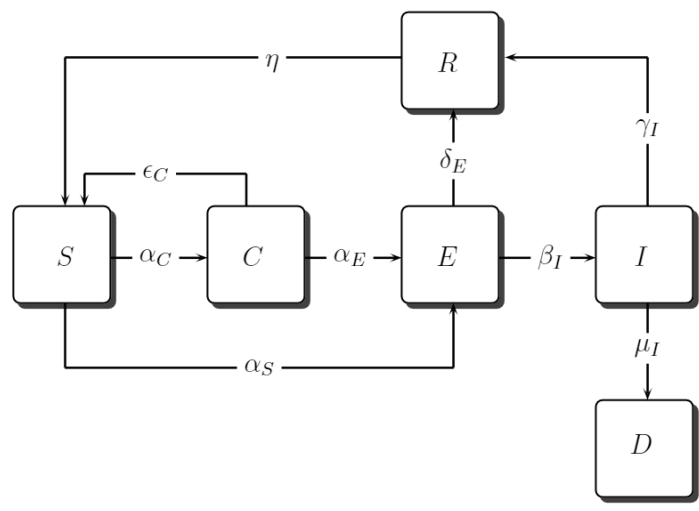

Figure 4: Diagram for SCEIRD model

Model parameters: $\alpha_{C}$ : rate of susceptible individuals who adopt a social distancing regime; $\alpha_{E}$ : transmission coefficient from confined to infected individuals that contract the virus and become exposed individuals; $\alpha_{S}$ : transmission coefficient from susceptible to infected individuals that contract the virus and become exposed; $\varepsilon_{C}$ : rate of confined individuals that abandon confinement and become susceptible again; $\delta_{E}$ : recovery rate of exposed individuals; $\beta_{I}$ : rate of exposed individuals that become infected; $\gamma_{I}$ : rate of recovery of infected individuals; $\mu_{I}$ : death rate of infected individuals; and $\eta$ : rate of immunity loss. The initial conditions of the ODE systems are: $S(0)=$ total population of Brazil $-1, C(0)=0, E(0)=0, I(0)=1, R(0)=0$ and $D(0)=0$.

Again, we do not consider vital dynamics in this case, that is,

$$
S(t)+C(t)+E(t)+I(t)+R(t)+D(t)=N=\text { constant } .
$$


The SCEIRD model can be writen as:

$$
\begin{aligned}
\frac{d S(t)}{d t} & =\varepsilon_{C} C(t)-\alpha_{C} S(t)-\alpha_{S} S(t) I(t)+\eta R(t) ; \\
\frac{d C(t)}{d t} & =\alpha_{C} S(t)-\varepsilon_{C} C(t)-\alpha_{E} C(t) I(t) ; \\
\frac{d E(t)}{d t} & =\alpha_{S} S(t) I(t)+\alpha_{E} C(t) I(t)-\beta_{I} E(t)-\delta_{E} E(t) ; \\
\frac{d I(t)}{d t} & =\beta_{I} E(t)-\gamma_{I} I(t)-\mu_{I} I(t) ; \\
\frac{d R(t)}{d t} & =\gamma_{I} I(t)+\delta_{E} E(t)-\eta R(t) ; \\
\frac{d D(t)}{d t} & =\mu_{I} I(t) .
\end{aligned}
$$

The disease free equilibrium (DFE) is $\left(S_{0}, 0,0,0,0,0\right)$, according to the Next Generation Method (NGM for short) and using $x=(E, I)$, we have

$$
\begin{gathered}
\mathscr{F}(x)=\left(\begin{array}{c}
\alpha_{S} S I+\alpha_{E} C I \\
0
\end{array}\right), \mathscr{V}(x)=\left(\begin{array}{c}
\left(\beta_{I}+\delta_{E}\right) E \\
-\beta_{I} E+\left(\gamma_{I}+\mu_{I}\right) I
\end{array}\right), F=\left(\begin{array}{cc}
0 & \alpha_{S} S_{0} \\
0 & 0
\end{array}\right) \\
V=\left(\begin{array}{cc}
\beta_{I}+\delta_{E} & 0 \\
-\beta_{I} & \gamma_{I}+\mu_{I}
\end{array}\right), \quad F V^{-1}=\left(\begin{array}{cc}
\frac{\beta_{I} \alpha_{S} S_{0}}{\left(\beta_{I}+\delta_{E}\right)\left(\gamma_{I}+\mu_{I}\right)} & \frac{\alpha_{S} S_{0}}{\gamma_{I}+\mu_{I}} \\
0 & 0
\end{array}\right),
\end{gathered}
$$

and thus

$$
R_{0}=\frac{\beta_{I} \alpha_{S} S_{0}}{\left(\beta_{I}+\delta_{E}\right)\left(\gamma_{I}+\mu_{I}\right)}
$$

\subsection{The SCEAIRD Model}

This model has as one of its objectives the ability to study the effect of social distancing with individually protective methods and educational campaigns, besides individuals' decision to abandon distancing due to social and economic pressures. In this case, we consider the following classes of individuals: $S$ (Susceptible), $C$ (Confined - individuals under a social distancing regime), $E$ (Exposed - individuals who contract the virus, but do not yet infect others, nor manifest symptoms), $A$ (Asymptomatic), $I$ (Infected), $R$ (Recovered) and $D$ (Dead), see Figure 5. Observe that Exposed, Asymptomatic and Infected individuals are all infected, but in different compartmental classes, or sub-compartments.

Model parameters: $\alpha_{A}$ : transmission coefficient from asymptomatic to susceptible individuals that contract the virus and become exposed; $\alpha_{I}$ : transmission coefficient from infected to susceptible individuals that contract the virus and become exposed; $\alpha_{C}$ : rate of susceptible individuals that become confined; $\varepsilon_{C}$ : rate of confined individuals that decide to end their social distancing regime; $\beta_{A}$ : transmission coefficient from asymptomatic to confined individuals that contract the virus and become exposed; $\beta_{I}$ : transmission coefficient from infected to confined individuals that contract the virus and become exposed; $\delta_{A}$ : rate of exposed individuals that become 


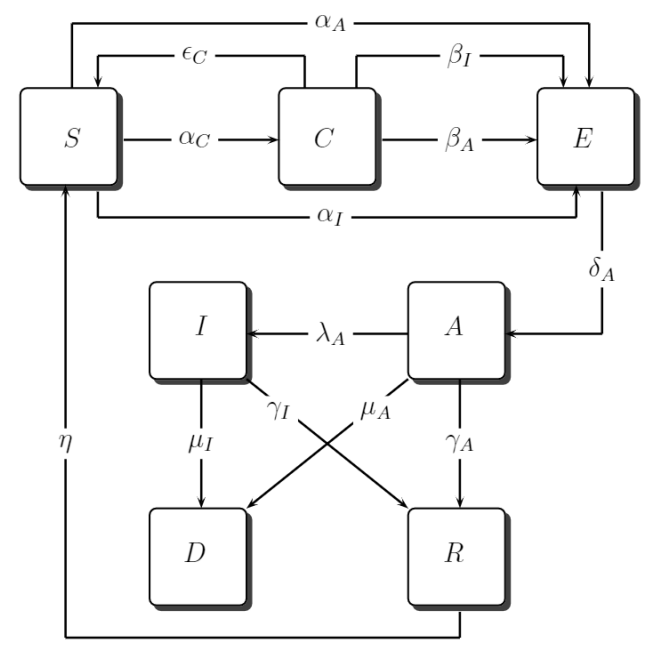

Figure 5: Diagram for SCEAIRD model

asymptomatic; $\lambda_{A}$ : rate of asymptomatic individuals that become infected when they start o show symptoms, test positive for COVID-19 and are counted in the infected number of a region; $\gamma_{A}$ : recovery rate of asymptomatic individuals; $\gamma_{I}$ : recovery rate of infected individuals; $\mu_{A}$ : death rate of asymptomatic individuals; $\mu_{I}$ : death rate of infected individuals; and $\eta$ : rate of immunity loss. The initial conditions of the ODE systems are: $S(0)=$ total population of Brazil $-1, C(0)=0$, $E(0)=0, A(0)=0, I(0)=1, R(0)=0$ and $D(0)=0$.

We do not consider vital dynamics in this model, that is,

$$
S(t)+C(t)+E(t)+A(t)+I(t)+R(t)+D(t)=N=\text { constant } .
$$

The SCEAIRD model can be written as:

$$
\begin{aligned}
\frac{d S(t)}{d t} & =\varepsilon_{C} C(t)-\alpha_{C} S(t)-\alpha_{A} S(t) A(t)-\alpha_{I} S(t) I(t)+\eta R(t) \\
\frac{d C(t)}{d t} & =\alpha_{C} S(t)-\varepsilon_{C} C(t)-\beta_{A} C(t) A(t)-\beta_{I} C(t) I(t) \\
\frac{d E(t)}{d t} & =\alpha_{A} S(t) A(t)+\alpha_{I} S(t) I(t)+\beta_{A} C(t) A(t)+\beta_{I} C(t) I(t)-\delta_{A} E(t) \\
\frac{d A(t)}{d t} & =\delta_{A} E(t)-\gamma_{A} A(t)-\lambda_{A} A(t)-\mu_{A} A(t) \\
\frac{d I(t)}{d t} & =\lambda_{A} A(t)-\gamma_{I} I(t)-\mu_{I} I(t) \\
\frac{d R(t)}{d t} & =\gamma_{A} A(t)+\gamma_{I} I(t)-\eta R(t) \\
\frac{d D(t)}{d t} & =\mu_{I} I(t)+\mu_{A} A(t) .
\end{aligned}
$$


The disease free equilibrium (DFE) is $\left(S_{0}, 0,0,0,0,0\right)$ and we used the Next Generation Method, explained in [18], to calculate the basic reproduction number. So let be $x=(E, A, I)$

$$
\begin{gathered}
\mathscr{F}(x)=\left(\begin{array}{c}
\alpha_{A} S A+\alpha_{I} S I+\beta_{A} C A+\beta_{I} C I \\
0 \\
0
\end{array}\right), \mathscr{V}(x)=\left(\begin{array}{c}
\delta_{A} E \\
-\delta_{A} E+\left(\gamma_{A}+\lambda_{A}+\mu_{A}\right) A \\
-\lambda_{A} A+\left(\gamma_{I}+\mu_{I}\right) I
\end{array}\right) \\
F=\left(\begin{array}{ccc}
0 & \alpha_{A} S_{0} & \alpha_{I} S_{0} \\
0 & 0 & 0 \\
0 & 0 & 0
\end{array}\right), \quad V=\left(\begin{array}{ccc}
\delta_{A} & 0 & 0 \\
-\delta_{A} & \gamma_{A}+\lambda_{A}+\mu_{A} & 0 \\
0 & -\lambda_{A} & \gamma_{I}+\mu_{I}
\end{array}\right), \\
F V^{-1}=\left(\begin{array}{ccc}
\frac{\left[\alpha_{I} \lambda_{A}+\alpha_{A}\left(\gamma_{I}+\mu_{I}\right)\right] S_{0}}{\left(\gamma_{I}+\mu_{I}\right)\left(\gamma_{A}+\lambda_{A}+\mu_{A}\right)} & \frac{\left[\alpha_{I} \lambda_{A}+\alpha_{A}\left(\gamma_{I}+\mu_{I}\right)\right] s_{0}}{\left(\gamma_{I}+\mu_{I}\right)\left(\gamma_{A}+\lambda_{A}+\mu_{A}\right)} & \frac{\alpha_{I} S_{0}}{\gamma_{I}+\mu_{I}} \\
0 & 0 & 0 \\
0 & 0 & 0
\end{array}\right) .
\end{gathered}
$$

Thus,

$$
R_{0}=\frac{\left[\alpha_{I} \lambda_{A}+\alpha_{A}\left(\gamma_{I}+\mu_{I}\right)\right] S_{0}}{\left(\gamma_{I}+\mu_{I}\right)\left(\gamma_{A}+\lambda_{A}+\mu_{A}\right)}
$$

\section{NUMERICAL RESULTS}

In this section, we present the numerical simulations of some scenarios in order to compare results of our model to the real-life data obtained from several reports published by World Health Organization (WHO) [20] and Worldometer [21] for the active cases of COVID-19 in Brazil.

We used a fourth order Runge-Kutta numerical method to solve our system of ordinary differential equation in Wolfram Mathematica. To run our model to Brazil, we used both coefficients estimated from outside sources and coefficients found by optimization methods.

The starting point of our simulations is January 1st, 2020 (day 1), but the Chinese authorities informed about the new virus only four days after the first case of COVID-19 was diagnosed on February 27th, 2020 in São Paulo. Here, we follow the data of the daily reports published by [21] and we show that our models describe in different ways the real data of daily confirmed cases during the 8 months of the outbreak. Furthermore, we numerically simulate the behaviour of the COVID-19 for the following two years, including a possible second wave and an endemic equilibrium of the disease.

According to the population census of 2019, the total population of Brazil is about 211 million people [4]. During the COVID-19 outbreak, there is a restriction of movements of individuals, in order to bring about a reduction of the spread of the disease. To all models, for the initial conditions, the following values have been fixed: $S(0)=211893786, C(0)=E(0)=A(0)=$ $R(0)=D(0)=0$ and $I(0)=1$. Others parameter values were estimated by curve fitting in the entire time domain. 


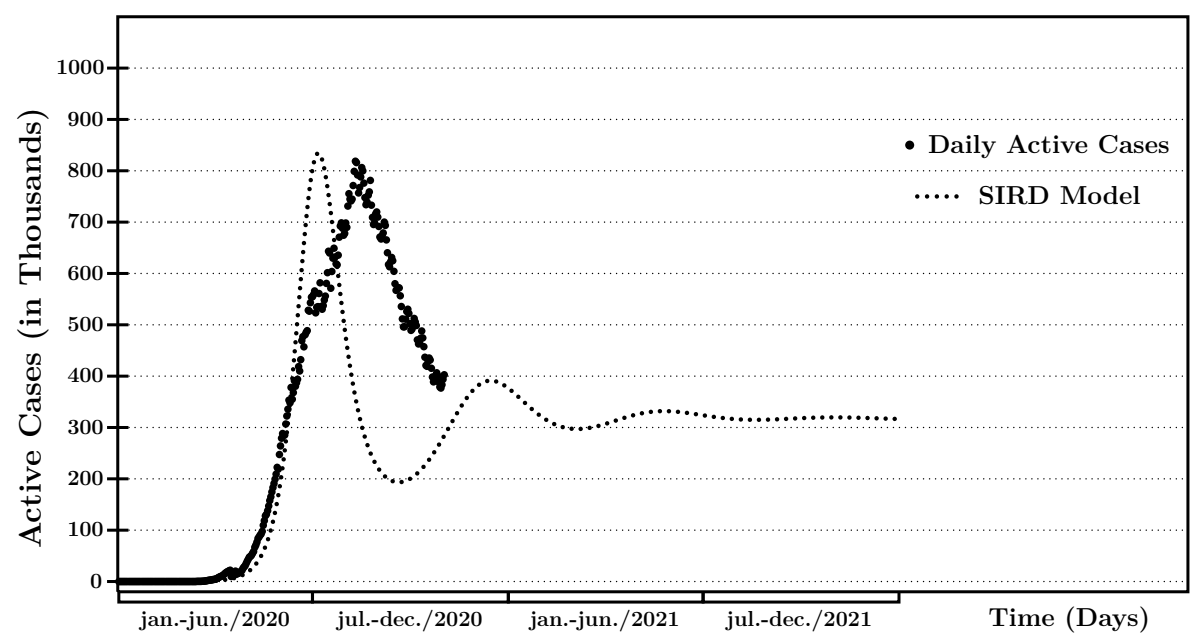

Figure 6: Active cases of COVID-19 in Brazil. This scenario was generated using data from [20] and the presented classic SIRD model with parameters: $\alpha_{S}=4.96 \times 10^{-9}$ (person $^{-1}$ day $\left.^{-1}\right)$ and $\eta=0.02\left(\right.$ day $\left.^{-1}\right)$, both estimated, and $\gamma_{I}=0.97\left(\right.$ day $\left.^{-1}\right)$ and $\mu_{I}=0.03\left(\right.$ day $\left.^{-1}\right)$ from [21]. In order to obtain coherent values for unknown parameters, the solutions were adjusted to the existing data provided in [21]. The estimate of $R_{0}$ obtained is 1.08 .

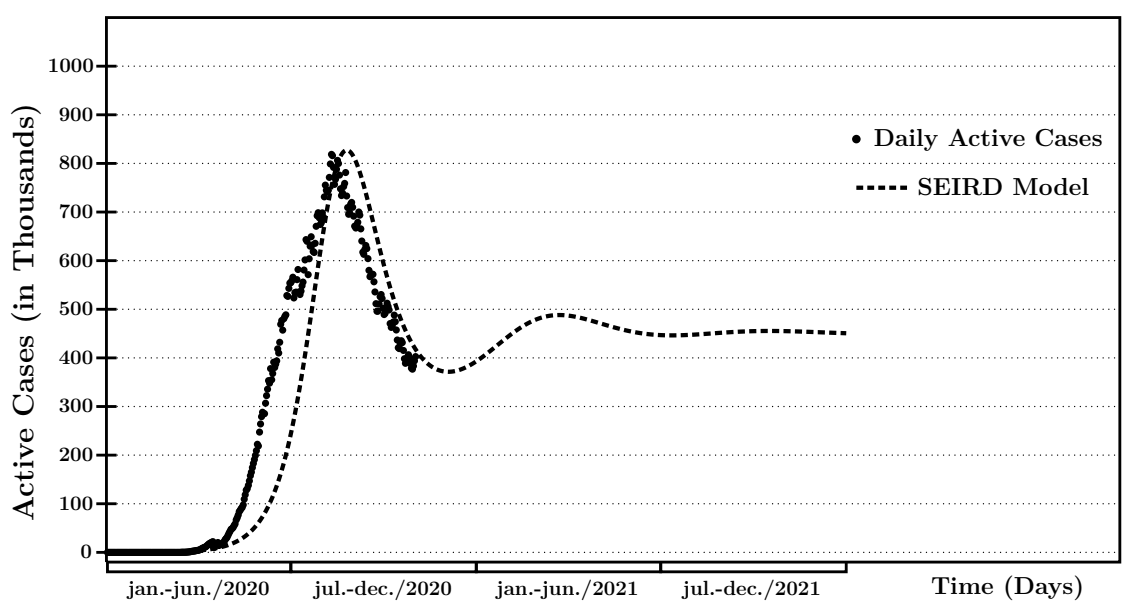

Figure 7: Active cases of COVID-19 in Brazil. This scenario was generated using data from [20] and the proposed SEIRD model with parameters: $\alpha_{S}=5.16 \times 10^{-9}\left(\right.$ person $^{-1}$ day $\left.^{-1}\right)$, $\alpha_{E}=0.65\left(d_{a y}^{-1}\right)$ and $\eta=0.02\left(d a y^{-1}\right)$, which were estimated, and $\gamma_{I}=0.97\left(\right.$ day $\left.^{-1}\right)$ and $\mu_{I}=0.03\left(d_{a y}{ }^{-1}\right)$ from [21]. In order to obtain coherent values for unknown parameters, the solutions were adjusted to the existing data provided in [21]. The estimate of $R_{0}$ is 1.13 . 


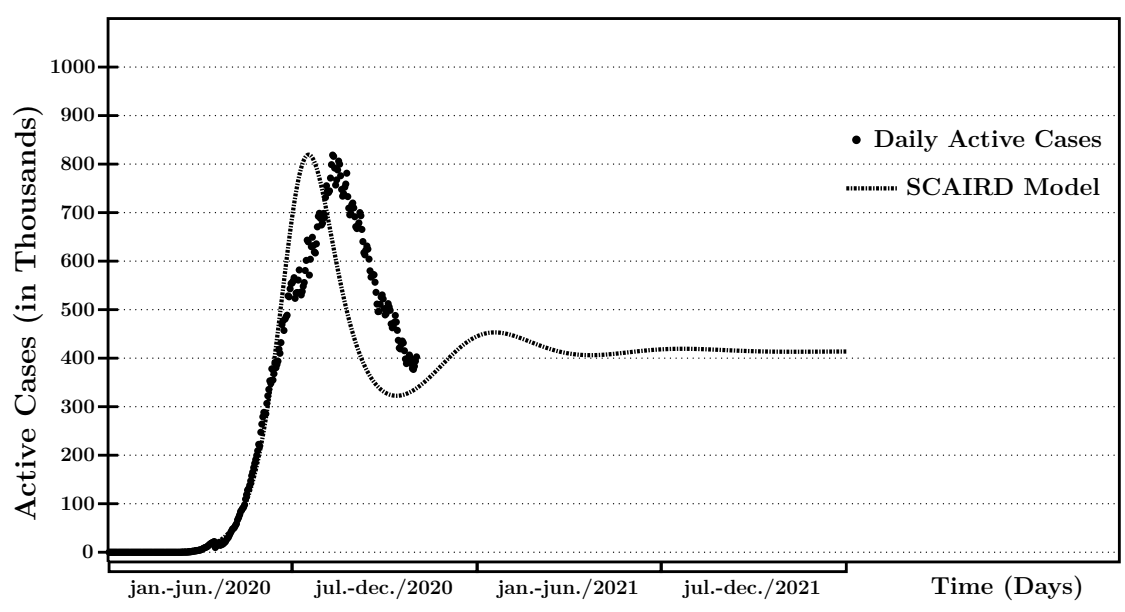

Figure 8: Active cases of COVID-19 in Brazil. This scenario was generated using data from [20] and the proposed SCAIRD model with parameters: $\alpha_{A}=4.71 \times 10^{-9}$ (person $^{-1}$ day $\left.^{-1}\right)$, $\alpha_{I}=7.16 \times 10^{-9}\left(\right.$ person $^{-1}$ day $\left.^{-1}\right), \alpha_{C}=0.4\left(\right.$ day $\left.^{-1}\right), \beta_{A}=4.71 \times 10^{-10}$ (person $^{-1}$ day $\left.^{-1}\right)$, $\beta_{I}=2.83 \times 10^{-9}\left(\right.$ person $^{-1}$ day $\left.^{-1}\right), \varepsilon_{C}=0.7\left(\right.$ day $\left.^{-1}\right), \gamma_{A}=0.4\left(\right.$ day $\left.^{-1}\right), \delta_{A}=0.5\left(\right.$ day $\left.^{-1}\right), \mu_{A}=$ $0.003\left(\right.$ day $\left.^{-1}\right)$ and $\eta=0.02\left(\right.$ day $\left.^{-1}\right)$, which were estimated, and $\tau=5.2($ day $), \gamma_{I}=0.97\left(\right.$ day $\left.^{-1}\right)$ and $\mu_{I}=0.03\left(d_{a y}{ }^{-1}\right)$ from [21]. In order to obtain coherent values for unknown parameters, the solutions were adjusted to the existing data provided in [21]. The estimate of $R_{0}$ is 1.80 .

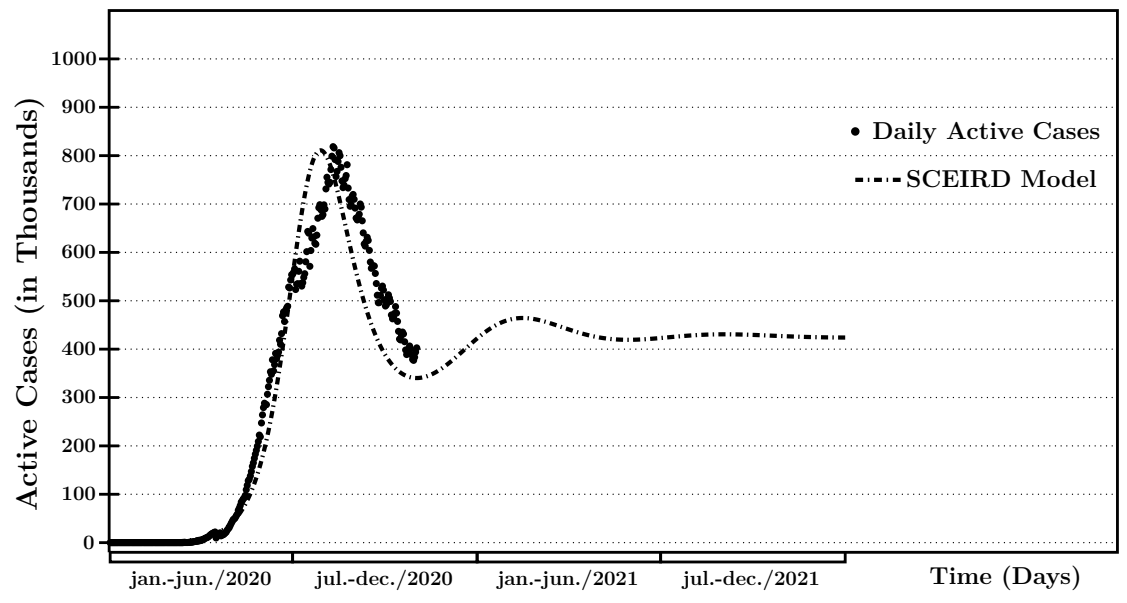

Figure 9: Active cases of COVID-19 in Brazil. This scenario was generated using data from [20] and the proposed SCEIRD model with parameters: $\alpha_{S}=7.35 \times 10^{-9}$ (person $^{-1}$ day $\left.^{-1}\right)$, $\alpha_{E}=4.71 \times 10^{-9}\left(\right.$ person $^{-1}$ day $\left.^{-1}\right), \alpha_{C}=0.4\left(\right.$ day $\left.^{-1}\right), \varepsilon_{C}=0.6\left(\right.$ day $\left.^{-1}\right), \beta_{I}=0.5\left(\right.$ day $\left.^{-1}\right)$, $\delta_{E}=0.1\left(d a y^{-1}\right)$ and $\eta=0.02\left(d a y^{-1}\right)$, which were estimated, and $\gamma_{I}=0.97\left(\right.$ day $\left.^{-1}\right)$ and $\mu_{I}=0.03\left(\right.$ day $\left.^{-1}\right)$ from [21]. In order to obtain coherent values for unknown parameters, the solutions were adjusted to the existing data provided in [21]. The estimate of $R_{0}$ is 1.33 . 


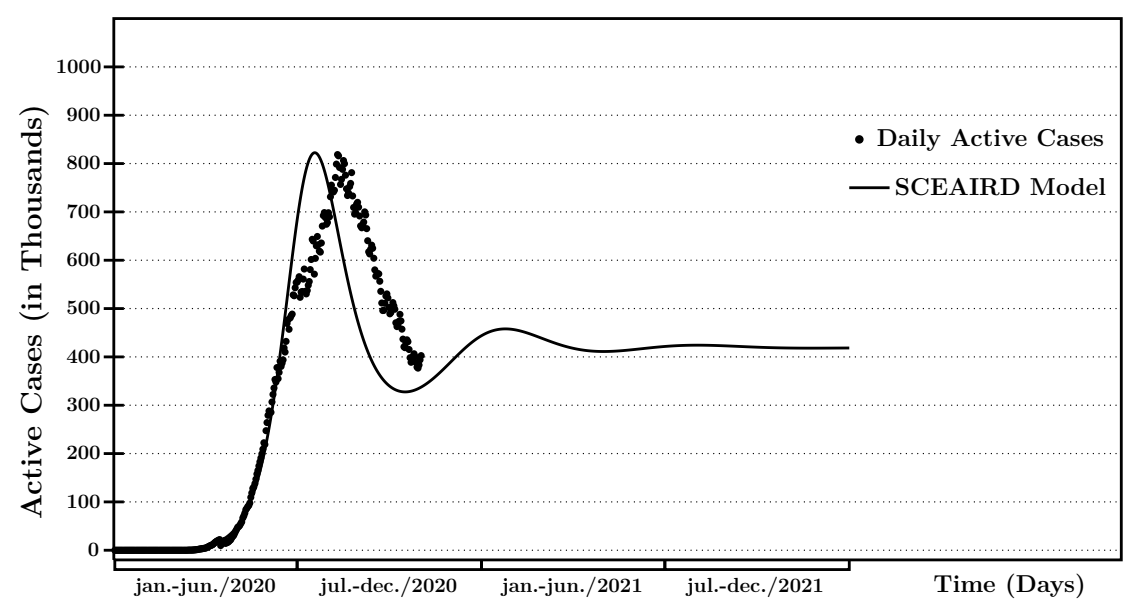

Figure 10: Active cases of COVID-19 in Brazil. This scenario was generated using data from [20] and the proposed SCEAIRD model with parameters: $\alpha_{A}=4.00 \times 10^{-9}\left(\right.$ person $^{-1}$ day $\left.^{-1}\right)$, $\alpha_{I}=6.36 \times 10^{-9}\left(\right.$ person $^{-1}$ day $\left.^{-1}\right), \quad \alpha_{C}=0.4\left(\right.$ day $\left.^{-1}\right), \quad \varepsilon_{C}=0.8\left(\right.$ day $\left.^{-1}\right), \quad \beta_{A}=1.88 \times$ $10^{-9}\left(\right.$ person $^{-1}$ day $\left.^{-1}\right), \beta_{I}=3.77 \times 10^{-9}\left(\right.$ person $^{-1}$ day $\left.^{-1}\right), \delta_{A}=0.4\left(\right.$ day $\left.^{-1}\right), \gamma_{A}=0.55\left(\right.$ day $\left.^{-1}\right)$, $\lambda_{A}=0.7\left(d a y^{-1}\right)$ and $\eta=0.02\left(d a y^{-1}\right)$, which were estimated, and $\gamma_{I}=0.97\left(\right.$ day $\left.^{-1}\right)$ and $\mu_{I}=0.03\left(\right.$ day $\left.^{-1}\right)$ from [21]. In order to obtain coherent values for unknown parameters, the solutions were adjusted to the existing data provided in [21]. The estimate of $R_{0}$ is 1.46 .

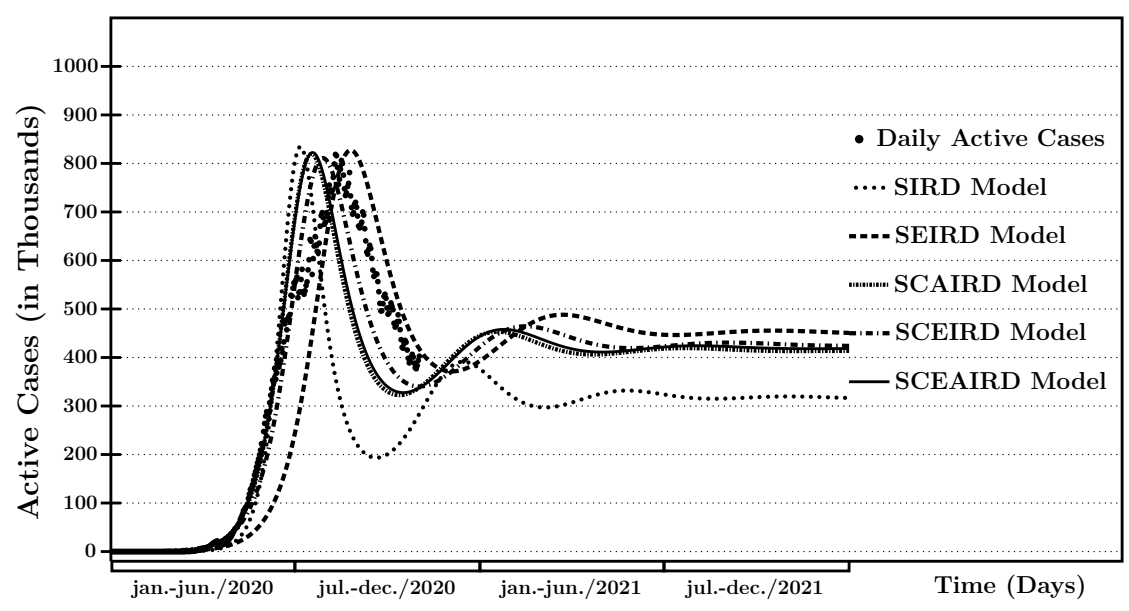

Figure 11: Active cases of COVID-19 in Brazil and the comparison between the models presented in this work. 


\section{FINAL COMMENTS}

In this paper we presented five different approaches to the modelling of the COVID-19 pandemic. All the models suppose the existence of at least four classes of individuals, namely: Susceptible, Infected, Recovered and Dead. We calculated the basic reproductive number associated to each model and obtained numerical simulations for real-life data in Brazil.

The numerical simulations show that the qualitative behaviour of the models is in its majority, similar. In fact, all the models presented predict a second wave of the infection, smoother than the first one. This can be attributed to the considered temporary immunity (albeit still under thorough investigation) of COVID-19, besides, when the loss immunity rate of the recovered individuals is zero, the numerical simulations does not predict a second wave of infections. Nevertheless, in many real situations, the so-called second wave being of greater magnitude than the first one [21], and considering studies [2] have indicated that changes in behaviours, treatments, social attitudes and pressures affect the used parameters, here considered, as in classical situations, constant.

The basic reproduction numbers in Brazil, according to [15], is in a range between 0,95 and 1,08. However, the values of the parameters used for the simulations of the models resulted in slightly higher values for $R_{0}$. This indicates that the estimation of the parameters must be improved and considered as varying in time, so that the simulations may present more realistic results. Realistic in the sense of describing radical changes in social behaviour and, consequently, contagion - and deaths.

Another reason for the difficulties encountered are related to the sub-notifications in all of Brazil [6] and, for the authors, adjusting the parameters without taking this into account introduces further distance from real-life data.

The content presented in this paper is the beginning of a better developed work concerning the modelling of COVID-19 in Brazil. Using both qualitative analysis and numerical simulations, the main objective of our research group is to obtain solutions to our models that satisfactorily represent the behaviour of the real data of Brazil cases and deaths and then try to understand the dynamics of is disease according to the assumptions considered herein.

Finally, the challenge of working with non-autonomous systems is immediate, since, as mentioned here, social, as well as economical (and, quite probably psychological as well) attitudes affect some of the models' parameters evolution, limiting the efficiency of the models beyond that of simulating scenarios for strategic decisions regarding public policies and possibilities for vaccination programmes.

\section{Acnowledgments}

The authors would like to thank Juliana Marta Rodrigues de Souza, Tiago Yuzo Miyaoka, Anny Almeida, José Eduardo Jesus da Silva and Marina de Souza Ripper that made significant contributions to the development of this work as well as the Institute of Mathematics, Statistics and 
Scientific Computing of the State University of Campinas for support in the development of this work.

Grantee CAPES 88887.369830/2019-00, CNPq 142309/2019-2, 140692/2020-7, Federal Institute of Alagoas.

\section{REFERENCES}

[1] H.M. Ashour, W.F. Elkhatib, M. Rahman, H.A. Elshabrawy et al. Insights into the recent 2019 novel coronavirus (SARS-CoV-2) in light of past human coronavirus outbreaks. Pathogens, 9(3) (2020), 186.

[2] G.C. Calafiore, C. Novara \& C. Possieri. A time-varying SIRD model for the COVID-19 contagion in Italy. Annual Reviews in Control, 50 (2020), 361-372.

[3] M. Cascella, M. Rajnik, A. Cuomo, S.C. Dulebohn \& R. Di Napoli. Features, evaluation and treatment coronavirus (COVID-19). In “Statpearls [internet]”. StatPearls Publishing (2020).

[4] I.B. de Geografia e Estatística IBGE. Projeção da população do Brasil e das Unidades da Federação. URL https://www.ibge.gov.br/apps/populacao/projecao/. [accessed 25-July-2020].

[5] P.L. Delamater, E.J. Street, T.F. Leslie, Y.T. Yang \& K.H. Jacobsen. Complexity of the basic reproduction number (R0). Emerging infectious diseases, 25(1) (2019), 1.

[6] G1-Globo. Brasil provavelmente tem mais casos de Covid-19 do que os relatados, afirma diretor da OMS. URL https://g1.globo.com/bemestar/coronavirus/noticia/2020/06/ 22/brasil-provavelmente-tem-mais-casos-de-covid-19-do-que-os-relatadosafirma-diretor-da-oms . ghtml. [accessed 26-January-2021].

[7] Y.R. Guo, Q.D. Cao, Z.S. Hong, Y.Y. Tan, S.D. Chen, H.J. Jin, K.S. Tan, D.Y. Wang \& Y. Yan. The origin, transmission and clinical therapies on coronavirus disease 2019 (COVID-19) outbreak-an update on the status. Military Medical Research, 7(1) (2020), 1-10.

[8] J.M. Heffernan, R.J. Smith \& L.M. Wahl. Perspectives on the basic reproductive ratio. Journal of the Royal Society Interface, 2(4) (2005), 281-293.

[9] W.O. Kermack \& A.G. McKendrick. A Contribution to the Mathematical Theory of Epidemics. Proceedings of the Royal Society of London. Series A, Containing Papers of a Mathematical and Physical Character, 115(772) (1927), 700-721. URL http://www. jstor.org/stable/94815.

[10] A.J. Kucharski, T.W. Russell, C. Diamond, Y. Liu, J. Edmunds, S. Funk, R.M. Eggo, F. Sun, M. Jit, J.D. Munday et al. Early dynamics of transmission and control of COVID-19: a mathematical modelling study. The lancet infectious diseases, (2020).

[11] G. Medema, L. Heijnen, G. Elsinga, R. Italiaander \& A. Brouwer. Presence of SARS-Coronavirus-2 in sewage. MedRxiv, (2020).

[12] S. Meo, A. Alhowikan, T. Al-Khlaiwi, I. Meo, D. Halepoto, M. Iqbal, A. Usmani, W. Hajjar \& N. Ahmed. Novel coronavirus 2019-nCoV: prevalence, biological and clinical characteristics comparison with SARS-CoV and MERS-CoV. Eur Rev Med Pharmacol Sci, 24(4) (2020), 2012-2019. 
[13] C.N. Ngonghala, E. Iboi, S. Eikenberry, M. Scotch, C.R. MacIntyre, M.H. Bonds \& A.B. Gumel. Mathematical assessment of the impact of non-pharmaceutical interventions on curtailing the 2019 novel Coronavirus. Mathematical Biosciences, (2020), 108364.

[14] G. Orive, U. Lertxundi \& D. Barcelo. Early SARS-CoV-2 outbreak detection by sewage-based epidemiology. Science of The Total Environment, (2020), 139298.

[15] I.C.C.. response team. Short-term forecasts of COVID-19 deaths in multiple countries. URL https://mrc-ide.github.io/covid19-short-term-forecasts/index.html\#authors. [accessed 17-August-2020].

[16] H.A. Rothan \& S.N. Byrareddy. The epidemiology and pathogenesis of coronavirus disease (COVID19) outbreak. Journal of autoimmunity, (2020), 102433.

[17] Y. Shi, Y. Wang, C. Shao, J. Huang, J. Gan, X. Huang, E. Bucci, M. Piacentini, G. Ippolito \& G. Melino. COVID-19 infection: the perspectives on immune responses (2020).

[18] P. Van den Driessche \& J. Watmough. Reproduction numbers and sub-threshold endemic equilibria for compartmental models of disease transmission. Mathematical biosciences, 180(1-2) (2002), 29-48.

[19] T.P. Velavan \& C.G. Meyer. The COVID-19 epidemic. Tropical medicine \& international health, 25(3) (2020), 278.

[20] W.H.O. WHO. Coronavirus disease (COVID-19) - Situation Report - 204. URL https : //covid19. who. int. [accessed 04-November-2020].

[21] Worldometers. Coronavirus incubation period. URL https://www.worldometers.info/ coronavirus/coronavirus-incubation-period. [accessed 04-November-2020]. 